\title{
Effect of the drift field on avalanche gain and charge collection in microgap detectors at high pressure
}

\author{
F.A.F. Fraga ${ }^{a}$ *, R. Ferreira Marques $^{\mathrm{a}}$, J.A.C. Gonçalves ${ }^{\mathrm{c}}$, J.R. Gonçalo ${ }^{\mathrm{a}}$, \\ A.J.P.L Policarpo ${ }^{\mathrm{a}}$, C.W.E. van Eijk ${ }^{\mathrm{b}}$, R.W. Hollander ${ }^{\mathrm{b}}$, F. van den Berg ${ }^{\mathrm{b}}$ \\ ${ }^{\text {a } L I P-C o i m b r a}$ and Departamento de Física da Universidade de Coimbra, 3000 Coimbra, Portugal \\ ${ }^{\mathrm{b}}$ Radiation Technology Group, IRI, Mekelweg 15, NL-2629 JB Delft, The Netherlands \\ ' IPEN and Departamento de Física, Pontíficia Universidade Católica, São Paulo, Brazil
}

\begin{abstract}
One of the main drawbacks of the use of microgap structures when used at high pressure ( 6 bar) at high count rates $\left(\sim 10^{6} \mathrm{~s}^{-1} \mathrm{~mm}^{-2}\right)$ is the limited maximum safe gain at which they can be operated. The use of a preamplification device such as the GEM (gas electron multiplier) can overcome this limitation; however, secondary space charge effects due to charge accumulation at the non-metallic surfaces cannot be neglected and should be minimised. These positive charges are due to the intrinsic multiplication of the GEM device and also to the drift of ions from the anode of the main detector. In this study we present data on the variation of the positive ion collection by the drift electrode versus drift field at several pressures up to 6 bar using a $\mathrm{Kr}-\mathrm{CO}_{2}$ mixture. Data were collected with microgaps having several anode and insulator widths. The influence of the drift field in the pulse rise time is also considered. (C) 1998 Elsevier Science B.V. All rights reserved.
\end{abstract}

\section{Introduction}

Microstrip and microgap gaseous detectors operated at high pressure have been considered for the development of 2D devices with low granularity, efficient in an energy range of up to $25 \mathrm{keV}$ in detectors for medical applications and synchrotron radiation [1-3]. We have reported that even when using microgaps with narrow anodes $(4 \mu \mathrm{m})$ the

\footnotetext{
* Corresponding author.; Fax: + 35139 29158; e-mail: francisco@filip3.fis.uc.pt.
}

safe maximum gain at 6 bar at high rates is low (under 200) [4] and that in order to be used in practical detectors these devices should be used with a preamplification structure such as the recently developed gas electron multiplier (GEM) [5]. It is known that space charge effects are important to proper GEM operation and that, in order to avoid recombination at high pressure, the typical drift fields are much higher than at atmospheric pressure. Considering the great interest of those preamplifying structures we decided to study the dependence of the charge collection on the drift electrode at several pressures using gaseous mixtures containing heavy gases such as krypton at 
various counting-rates and gains. Since our previous work has shown that the anode and insulator width is of great importance for high-pressure operation of microgaps, their influence on the drift current is also considered.

\section{System description}

The experimental system used was similar to the one already described in Refs. [3,4] and therefore only some details will be given. A stainless-steel chamber and a clean gas system were used. Special care was taken to keep the gas impurities to a minimum. Two series connected picoammeters (Keithley 602 and 414s) were used for simultaneous measurement of the anode and cathode currents. The drift electrode current was computed from the difference between these two values. Considering the very low currents, special care was taken to insulate the floating picoammeter in order to minimise leakage currents and a separate electrostatic shield was used to lower the induced noise during the measurements. The distance between the microgap surface and the drift electrode was $5 \mathrm{~mm}$.

The microgaps, that were produced by the Delft University of Technology, were built on a substrate of silicon covered with a thermally grown oxide layer [4]. The electrodes were made of aluminium and the insulator is silicon oxide of $3 \mu \mathrm{m}$ thickness. The anode pitch is $100 \mu \mathrm{m}$ and several anode and insulator widths were used as described in Table 1 . The useful area of each section of the microgap is $1 \times 8 \mathrm{~mm}^{2}$. The measurements were performed with a beam energy of $12 \mathrm{keV}$ using an X-ray generator. The beam was collimated to $0.5 \times 3 \mathrm{~mm}^{2}$ and carefully focused on the central part of the active structure under study to avoid border effects. The gain was calibrated either by the pulse amplitudes or by the current and count rate measurements.

\section{Experimental measurements}

During all the measurements the gas mixture was $\mathrm{Kr}-10 \% \mathrm{CO}_{2}$ and the gain was set at 150 within an error of less than $10 \%$ at a drift voltage of $1000 \mathrm{~V}$. The anode voltage was kept fixed when the drift
Table 1

Parameters of the microgap plates
Substrate material

Electrode metal

Insulator material

Anode strip height

Insulator strip height

Cathode strip height

Anode pitch
silicon
aluminium
silicon oxide
$1.0 \mu \mathrm{m}$
$3.0 \mu \mathrm{m}$
$0.6 \mu \mathrm{m}$
$100 \mu \mathrm{m}$

Combinations of anode strip widths $\left(\mathrm{W}_{\mathrm{s}}\right)$ and insulator widths $\left(\mathrm{W}_{\mathrm{i}}\right)(\mu \mathrm{m})$

\begin{tabular}{rrrrrrrrr}
\hline $\mathrm{W}_{\mathrm{s}}$ & 3 & 5 & 5 & 5 & 7 & 7 & 7 & 10 \\
$\mathrm{~W}_{\mathrm{i}}$ & 10 & 15 & 20 & 25 & 12 & 20 & 25 & 30 \\
\hline
\end{tabular}

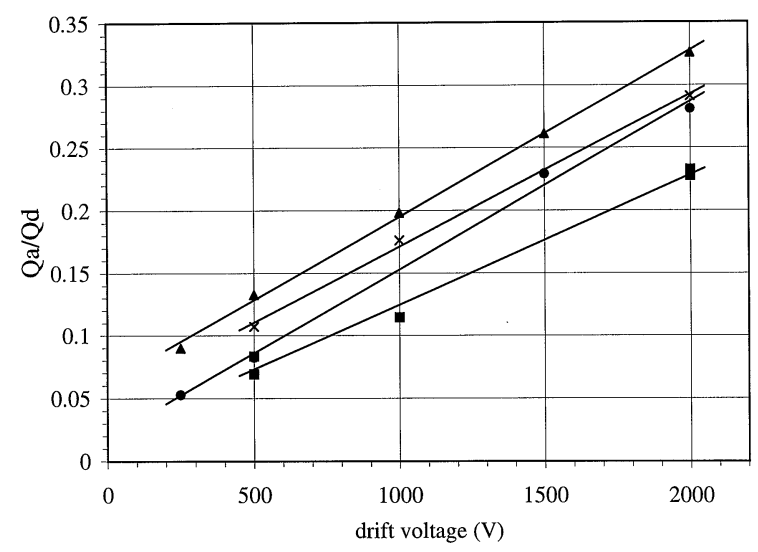

Fig. 1. Ratio between the drift and the anode currents versus drift voltage for two anode layouts $(3$ and $10 \mu \mathrm{m}$ anode widths, with 10 and $25 \mu \mathrm{m}$ insulator widths, respectively) and two counting rates $\left(1.6 \times 10^{5}\right.$ and $1.6 \times 10^{6}$ counts $\left.\mathrm{mm}^{2} \mathrm{~s}^{-1}\right)$. The mixture was $\mathrm{Kr}-10 \% \mathrm{CO}_{2}$ at 6 bar. (ム) $5 \mu \mathrm{m}$ anodes, $1.6 \times 10^{5} \mathrm{~Hz} \cdot \mathrm{mm}^{-2} ;\left(\mathbf{)} 5 \mu \mathrm{m}\right.$ anodes, $1.6 \times 10^{6} \mathrm{~Hz} \cdot \mathrm{mm}^{-2} ;(\times)$ $3 \mu \mathrm{m}$ anodes, $1.6 \times 10^{5} \mathrm{~Hz} \cdot \mathrm{mm}^{-2}$; (ם) $3 \mu \mathrm{m}$ anodes, $1.6 \times 10^{6}$ $\mathrm{Hz} \cdot \mathrm{mm}^{-2}$.

voltage was varied. As reported before, the change in gain when the drift voltage is doubled is less than $15 \%$ [4].

As expected, the drift current also depends on the value of the drift voltage. Fig. 1 shows the ratio of the drift and anode currents for two count rates and two different anode layouts versus applied drift voltage. When using the wider anodes and insulators the anode voltage is higher than with the narrower ones [4], and this can explain the observed difference in drift currents. At high counting 
rates less charge flows to the drift electrode. No significant reduction in gain was measured at the maximum used counting rate at a gain of $\sim 200$ [4].

The variation of the ratio between the drift and anode current with the counting rate is shown for several drift voltages in Fig. 2. The reduction of this ratio at the maximum counting rate (about 5\%) seems almost independent of drift voltage.

Fig. 3 shows the variation of the ratio between the drift and anode current versus counting rate

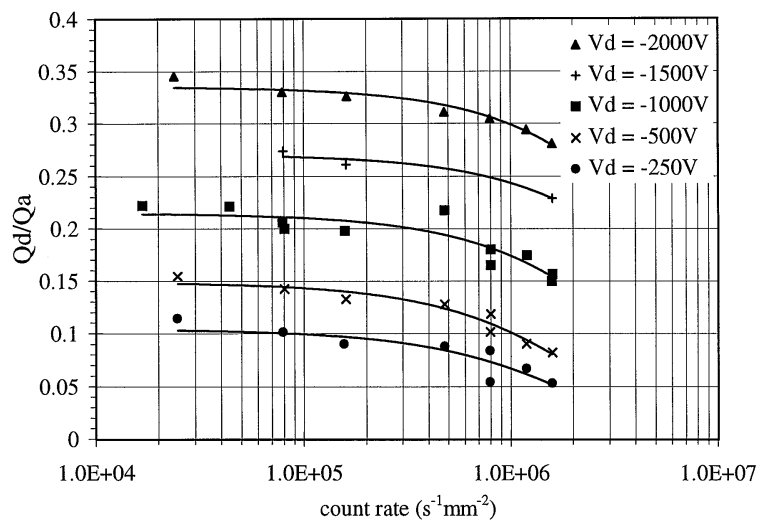

Fig. 2. Ratio between the drift and the anode currents versus counting rate for several values of drift voltage. The anode and insulator widths were 5 and $25 \mu \mathrm{m}$. The mixture was $\mathrm{Kr}-10 \% \mathrm{CO}_{2}$ at 6 bar and anode voltage was $452 \mathrm{~V}$.

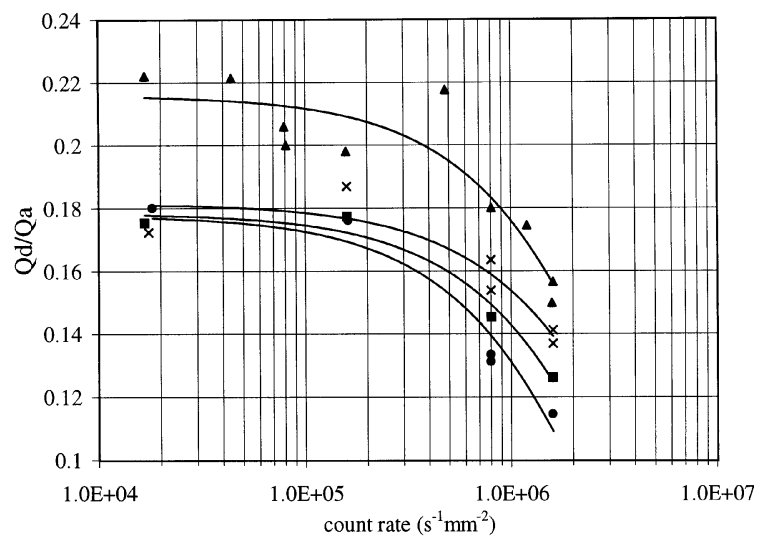

Fig. 3. Ratio between the drift and the anode currents versus counting rate for several anode layouts (anode width of $5 \mu \mathrm{m}$ and insulator widths of $15 \mu \mathrm{m}(\boldsymbol{\square}), 20 \mu \mathrm{m}(\times)$ and $25 \mu \mathrm{m}(\boldsymbol{\Delta})$; anode width of $3 \mu \mathrm{m}$ and insulator width $10 \mu \mathrm{m}(\mathbf{O}))$. The mixture was $\mathrm{Kr}-10 \% \mathrm{CO}_{2}$ at 6 bar. using four different microgap layouts. The general trend of the preceding figures appears again, wider insulators yield higher drift currents and the reduction in drift current is not significantly dependent on the anode layout.

The fraction of the avalanche charge which is collected at the drift electrode versus counting rate for different values of pressure is shown in Fig. 4. At 3 bar the reduction in the ratios between drift and anode currents seems less evident than at 6 bar, but taking in to account the associated measuring errors this reduction cannot be considered significantly different. At 1 bar the variation is smaller, being almost non-existent.

Considering that the rise time of the anode pulses depends mainly on the collection time of the positive ions of the avalanche, we made some measurements of the rise time of the anode charge signals. This parameter is, of course, relevant for high counting rate applications. After deconvolution of the rise time of the preamplifier (10 ns) the intrinsic rise time of the anode pulse at 1 bar was typically 56 ns with a drift voltage of $166 \mathrm{~V}$. A $20 \%$ decrease in rise time was measured when the drift voltage was increased to $1000 \mathrm{~V}$. It should be noted that the $\mathrm{Kr}$ mixture shows slower pulses than the usually quoted values for the Ar-DME mixtures. At 6 bar the variation of the rise time of the anode signals

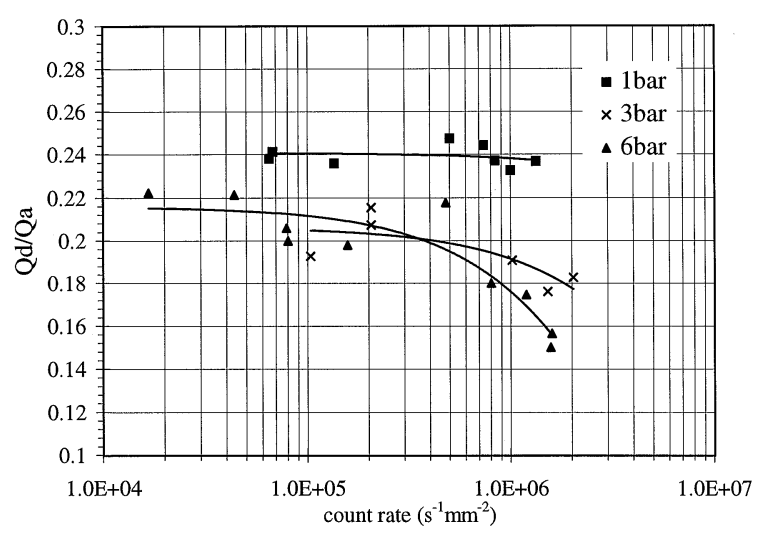

Fig. 4. Ratio between drift and anode currents versus counting rate at 1,3 and 6 bar with $V_{\mathrm{D}}=1000 \mathrm{~V}$. The microgap had 7 and $25 \mu \mathrm{m}$ anode and insulator widths at the 1 bar measurements and, 7 and $25 \mu \mathrm{m}$ anode and insulator widths at 3 and 6 bar. The mixture was $\mathrm{Kr}-10 \% \mathrm{CO}_{2}$. 


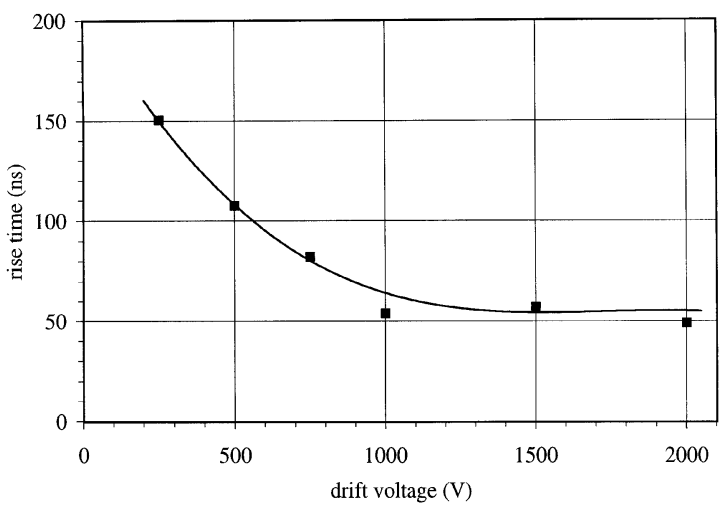

Fig. 5. Measured anode pulses rise time versus drift voltage. The mixture was $\mathrm{Kr}-10 \% \mathrm{CO}_{2}$ at 6 bar.

with the drift voltage is very clear and is shown in Fig. 5. The pulse rise time decreases with increasing drift voltage up to $1000 \mathrm{~V}$, reaching saturation at about 50 ns.

\section{Conclusions}

Our study of the drift current has shown that its value is strongly dependent on the value of the drift field and counting rate and that with the typical drift fields used at high-pressure operation the amount of positive ions that is collected by the drift electrode is important. While we could not see effects that could be easily associated exclusively with the influence of the layout on the avalanche development in the studied microgaps, it was shown that microgaps with wider insulating widths feature higher drift currents.

Considering the importance of the pulse speed in high count rate applications we have shown that microgap detectors at 6 bar should be operated with a sufficiently high drift field to have a fast rise time. However, in these conditions, as much as $20 \%$ of the avalanche ions are collected in the drift electrode. The influence of this charge on the proper operation of the GEM multiplying device at high pressure is still to be studied.

\section{References}

[1] S. E. Baru, V. V. Neusroev, A. Papanestis, G. A. Savinov, and L. I. Shekhtman, Proc. Int. Workshop on Micro-Strip Gas Chambers, Lyon, France, (1995), p. 27.

[2] F. Angelini, R. Bellazzini, A. Brez, M.M. Massai, G. Spandre, M. R. Torquati, Proc. European Workshop on X-Ray Detectors for Synchrotron Radiation Sources, Aussois, Frances, (1991), p. 96.

[3] F.A.F. Fraga, M.M.F.R. Fraga, R.F. Marques, L.M.S. Margato, J.R. Gonçalo, A.J.P.L. Policarpo, C.W.E. van Eijk, R.W. Hollander, J. van der Marel, Nucl. Instr. and Meth. (1997).

[4] F.A.F. Fraga, R. Ferreira Marques, I. Ivaniouchenkov, L.M.S. Margato, J.R. Gonçalo, A.J.P.L Policarpo, C.W.E. van Eijk, R.W. Hollander, J.van der Marel, presented at the IEEE 97 Nuclear Science Symp.

[5] R. Bouclier, W. Dominik, M. Hoch, J. C. Labbé, G. Million, L. Ropelewski, F. Sauli, CERN-PPE/97-32. 